\title{
Carrier Synchronization in Software Defined Radio using Costas Loop
}

\author{
A. Priya ${ }^{1 *}{ }^{*}$ N. Rajesh ${ }^{2}$ and R. Muthaiah ${ }^{3}$ \\ 1PG Student, School of Computing (VLSI), SASTRA University, 613401, \\ Thanjavur, Tamilnadu, India; sk.pri08@gmail.com \\ ${ }^{2}$ Associate Prof, School of EEE, SASTRA University, 613401, \\ Thanjavur, Tamilnadu, India; nrajesh@eee.sastra.edu \\ ${ }^{3}$ Professor, School of Computing (VLSI), SASTRA University, 613401, Thanjavur, Tamilnadu, India; \\ sjamuthaiah@core.sastra.edu
}

\begin{abstract}
This paper presents carrier synchronization in software defined radio for 8PSK technique. Software Defined Radio (SDR) plays a major solution for the need for flexibility, upgradability, and the problems of implementing multiple radio standards alternatively and even running several services in parallel. Previously carrier synchronization in software defined radio is implemented using QPSK technique and synchronization is done using PLL concept. The drawback in this we can transmit only few (only two) number of bits while synchronization is performed. The carrier synchronization by software defined radio using 8PSK technique will allow more number (three) of bits during synchronization, and the same bandwidth which is used for QPSK technique. The main advantage is the transmitting of three bits which reduces the time consumption and the synchronization is performed using COSTAS LOOP. The purpose and advantage of Costas loop compared to PLL is error voltage. The error voltage is less in Costas loop, due to this synchronization is performed effectively. The complete codings are coded using MATLAB.
\end{abstract}

Keywords: Software Defined Radio, 8PSK, Costas Loop.

\section{Introduction}

Software defined Radio is considered as an emerging technology on the communication industry. Most of the development based problems in Software defined radio is seen as a solution for elasticity, upgradability, and the challenges of implementing various radio standards alternatively. Nowadays in military radio applications and civilian radio applications, analog radios are replaced by digital radios. Using software techniques the digital signals are handled in SDR. Modifying radio devices cost-effectively has become business critical, and this is achieved by using SDR.

An antenna is used to select the signal from SDR. Then the analog signal is transformed into digital signal. Then in the software, the digital signal values are processed, and the output is converted into an audio signal or video signal.

In software defined radio the bandwidth of frequency, air interface protocol and functionality can be upgraded with the help of software techniques instead of hardware technique. Different types of modulation schemes are used for data transmission in SDR, such as QAM, BPSK, QPSK, 8PSK etc. $[3,4]$.

In this paper carrier synchronization in software defined radio is implemented using MATLAB and the sections are devised as follows: Section II discusses about proposed modulation scheme. Section III discusses about carrier synchronization. Section IV discusses about Costas loop. Section V discusses Performance Evaluation and eventually Section VI concludes the paper.

*Corresponding author:

A. Priya (sk.pri08@gmail.com) 


\section{8PSK Design Review}

In wireless communication industry eight phase shift keying (8PSK) is one type of phase modulation scheme. Three bits are encoded in one symbol, thereby increasing the data rate in the same bandwidth compared to QPSK. By using 8PSK more number of data is transmitted in less time. Eight phase shift keying or 8PSK uses eight phase-shifts (Figure 1). These occur at $\pi / 8,3 \pi / 8,5 \pi / 8,7 \pi / 8,9 \pi / 8,11 \pi / 8,13 \pi / 8$, and $15 \pi / 8$. Its phase difference is $\pi / 4$.

For 8PSK modulation the transmitted signal can be represented as

$$
\mathrm{S}_{\mathrm{i}}(\mathrm{t})=\sqrt{\frac{2 E}{T}} \cos \left(2 \pi \mathrm{f}_{\mathrm{c}} \mathrm{t}+(2 \mathrm{i}-1) \frac{\pi}{M}\right)
$$

where, $\mathrm{i}=1,2 \ldots \mathrm{M}$

The term $(2 i-1) \pi / M$ represents the phase of the signal. Here $M=8$, where $s_{i}(t)$ represents a possible state of sending $\mathrm{k}$ bits together. The signal can be represented as

$$
\mathrm{s}_{\mathrm{i}}(\mathrm{t})=\mathrm{s}_{\mathrm{i} 1} \Phi_{1}(\mathrm{t})+\mathrm{s}_{\mathrm{i} 2} \Phi_{2}(\mathrm{t}) \quad \text { for } \mathrm{i}=1,2 \ldots \mathrm{M} .
$$

$\Phi_{1}(\mathrm{t}) \& \Phi_{2}(\mathrm{t})$ defined as follows

$$
\begin{aligned}
& \Phi_{1}(\mathrm{t})=\sqrt{\frac{2}{T}} \cos \left(2 \pi \mathrm{f}_{\mathrm{c}} \mathrm{t}\right) \\
& \Phi_{2}(\mathrm{t})=\sqrt{\frac{2}{T}} \sin \left(2 \pi \mathrm{f}_{\mathrm{c}} \mathrm{t}\right)
\end{aligned}
$$

It can be easily seen that

$$
\begin{aligned}
\mathrm{S}_{\mathrm{i} 1} & =\sqrt{E} \cos \left((2 \mathrm{i}-1) \frac{\pi}{M}\right) \\
\mathrm{S}_{\mathrm{i} 2} & =-\sqrt{E} \sin \left((2 \mathrm{i}-1) \frac{\pi}{M}\right)
\end{aligned}
$$

To get $\mathrm{s}_{\mathrm{i}}(\mathrm{t})$ substitute $\Phi_{1}(\mathrm{t}), \Phi_{2}(\mathrm{t}), \mathrm{s}_{\mathrm{i} 1} \mathrm{~s}_{\mathrm{i} 2}$ in the signal equation.

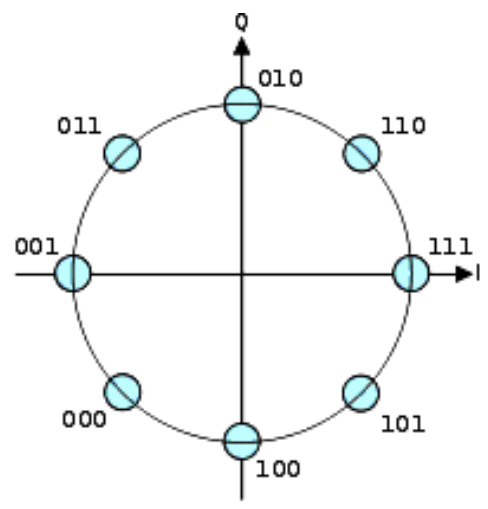

Figure 1. Constellation diagram of 8PSK.

\section{Carrier Synchronization}

In a digital communication different types of synchronization are established, which are carrier, frame and symbol synchronization. In wireless communication Carrier synchronization is considered as a general challenge. Different methods are there to implement the carrier synchronization in communication system. Phase locked loop, Costas loop are the types of method to achieve carrier synchronization. In this paper carrier synchronization is achieved by Costas loop. Local oscillator is required for both transmitter and receiver. The synchronization of the local oscillator at the reception side with the local oscillator at the source is carrier synchronization.

Due to reflection and refraction, the signal travels via different paths from the transmitter to receiver during transmission. This process is known as multipath effect (Figure 2) which affects the quality of the signal.

Due to multipath propagation error occurs in the signal. Errors are due to inter symbol interference which makes the communication less reliable. These interferences of one symbol with successive symbols can be minimized by using Raised cosine filter which is a type of pulse shaping filter.

Carrier synchronization is achieved by Costas loop. The Costas loop is used to remove the phase and frequency offsets in the receiver. Frequency instability in either the transmitter or receiver, and the Doppler Effect when the receiver is in motion relative to the transmitter are the sources of carrier frequency offset. Carrier phase offset is due to phase instability in oscillators and the noise $[1,2]$.

\section{Costas Loop}

Costas loop is used to recover the carrier phase from suppressed- carrier modulated signals. In wireless receivers Costas loop is used. When compared with the PLL based

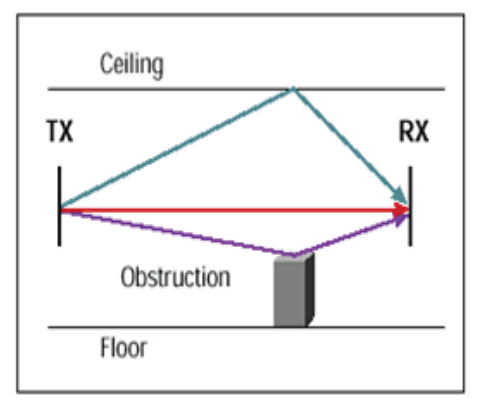

Figure 2. Multipath effect. 
detectors, the error voltage of Costas loop is $\sin \left(2\left(\theta_{i}-\theta_{f}\right)\right)$ vs $\sin \left(\theta_{i}-\theta_{f}\right)$ at small deviations. So the sensitivity is doubled and Costas loop is capable of tracking Doppler shifted carriers especially in OFDM and GPS receivers. Costas loop consists of two coherent detectors both are supplied with the demodulated signal. The detector in the upper path is referred as in-phase signal and the detector in the lower path is referred as quadrature-phase signal.

The basic principle behind the Costas receiver is that the two detectors together form a negative feedback system acting in a way to maintain the local oscillator synchronous with the incoming carrier wave. Let us consider two situations:

1. Phase synchronized between the incoming carrier wave and the local oscillator carrier: This is equivalent to $\theta=0$. In this case, the I-channel output contains the demodulated signal $\mathrm{m}(\mathrm{t})$. The Q-channel output is zero because of the quadrature null effect.

2. Loss of Phase synchronization between the incoming carrier wave and the local oscillator carrier: This is equivalent to $\theta \neq 0$. The I-channel output essentially remains unchanged. However, now, there will be some signal appearing at the Q-channel output. The amplitude of the signal is proportional to $\sin \theta$. If $\theta>0$, the $Q$ channel output will have the same polarity as the I-channel output. If $\theta<0$, the polarity of the Q-channel output is the negative of that of the I-channel output. The two channel outputs
$\mathrm{A}_{c} \mathrm{~m}(\mathrm{t}) \cos \theta$ and $\mathrm{A}_{c} \mathrm{~m}(\mathrm{t}) \sin \theta$ are provided as inputs to the multiplier followed by a low pass filter. The output of the multiplier is $\left(\mathrm{m}^{2}(\mathrm{t}) \sin 2 \theta\right) / 2$. The low pass filter following the multiplier is of a very narrow bandwidth. Therefore, it essentially generates a DC output, a value proportional to $\sin 2 \theta$. This is known as ksin2 $\theta$, at the input to the VCO in the Figure 3. This DC signal serves as a correction signal for the VCO. The VCO generates a carrier with the required phase correction. The system thus generates a carrier that is phase-locked to the incoming carrier wave [7].

\section{Performance Evaluation}

The 8PSK system includes following blocks: signal generator, interpolation, modulation, channel, demodulation, decimation, carrier recovery (Figure 4).

The input signal to the 8PSK modulation scheme is generated in signal generator (Figure 5). Next the input signal is interpolated.

The purpose of interpolation is to increase the sampling rate, so that the interpolated signal can be given as input to other systems which is operating at a higher sampling rate. For multi rate signal processing technique, and to get better performance of the system in terms of speed and resource interpolation is used. To enhance the bandwidth efficiency raised cosine filter is used (Figure 6). After the process of interpolation, modulation begins.

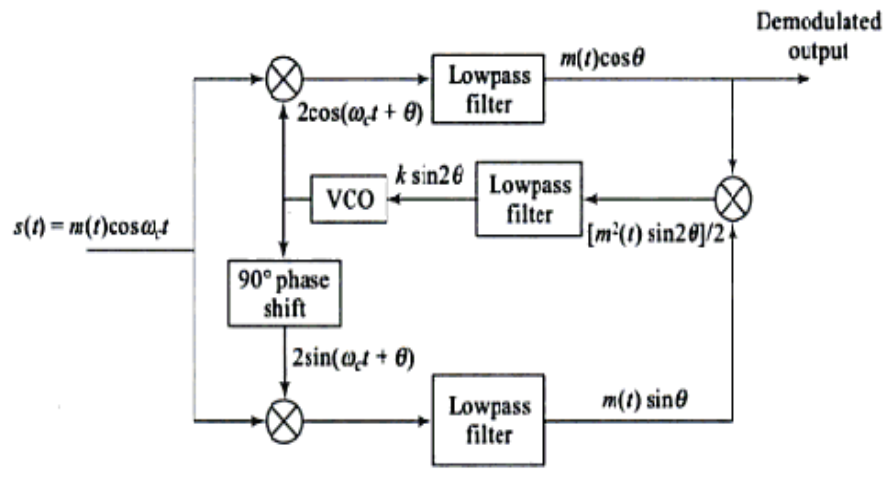

Figure 3. Basic Costas loop block diagram.

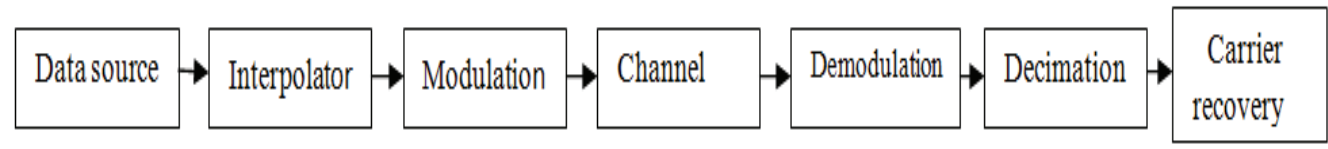

Figure 4. Block diagram. 
The process of mixing the message signal or the data signal with the carrier signal is known as modulation. The purpose of modulation is changing the data signal which is suitable for transmission. The carrier signal frequency taken at the transmitter side is $70 \mathrm{MHz}$ which is shown in Figure 7. For carrier synchronization the transmitter and receiver carrier signal frequencies must be synchronized. The modulated signal is shown in Figure 8.

The modulated signal is sent via the channel. Based on the data length of the signal, it gets affected by noise. Consider the data length as 6 and 8 for example. In this case Channel interference is more when the data length is 8 which are shown in the following Figures 9 and 10.

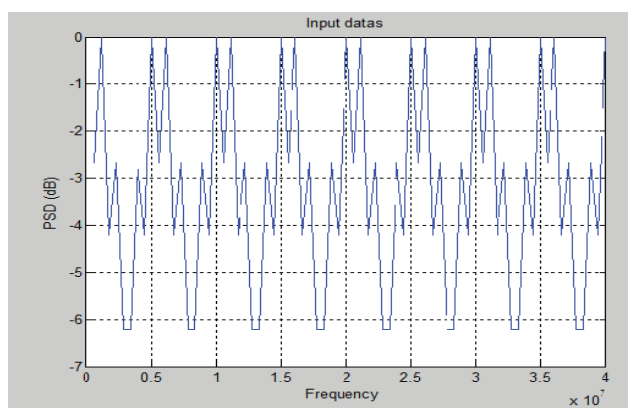

Figure 5. Input signal.

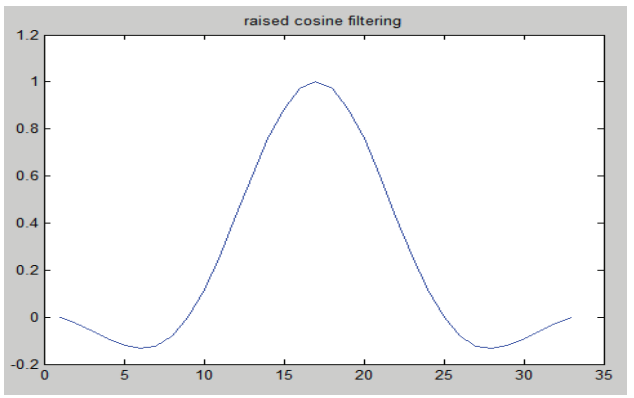

Figure 6. Raised cosine filtering.

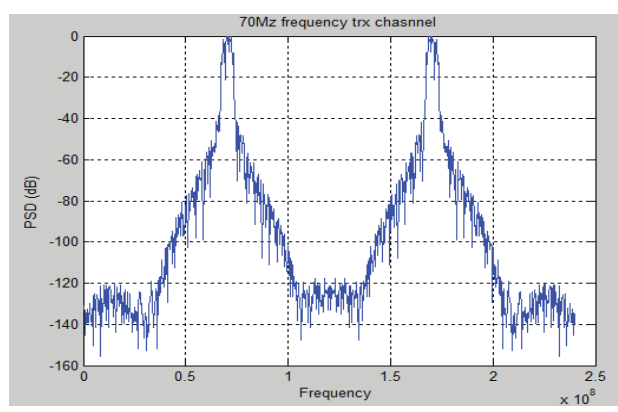

Figure 7. Carrier signal frequency in transmitter side.
Then the received signal is decimated for demodulation (Figure 11). Sampling rate is reduced in the decimation process. This process is followed by demodulation which is the reverse process of modulation. Carrier signal frequency taken at the receiver side is $10 \mathrm{MHz}$ (Figure 12).

Reflection and refraction of the signal from earthbound objects such as foothills and constructions is the main source for multipath effect. Due to multipath effect the phase of the signal will change, and it produce the interference. Due to interference fading will occur in the data signal. So to correctly recover the original data from the received signal carrier synchronization must be performed. Carrier synchronization is achieved by Costas loop which is used to

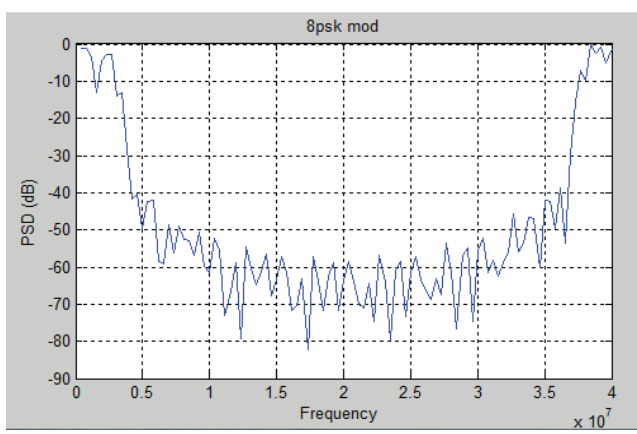

Figure 8. Modulated signal.

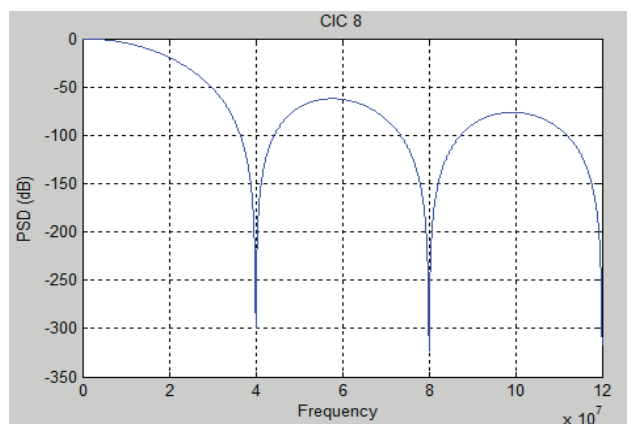

Figure 9. Coefficients of 8 - Channel interference.

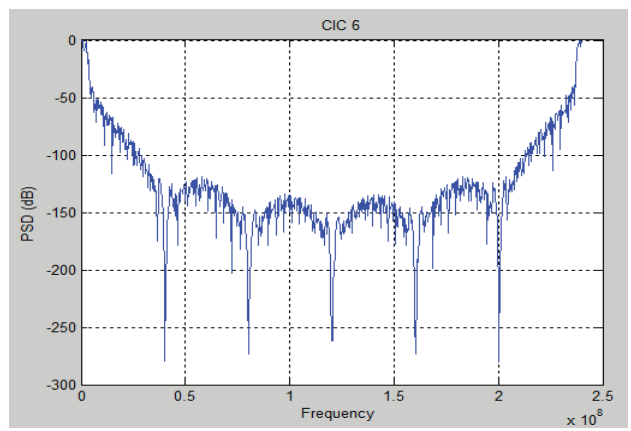

Figure 10. Coefficients of 6 - Channel interference. 


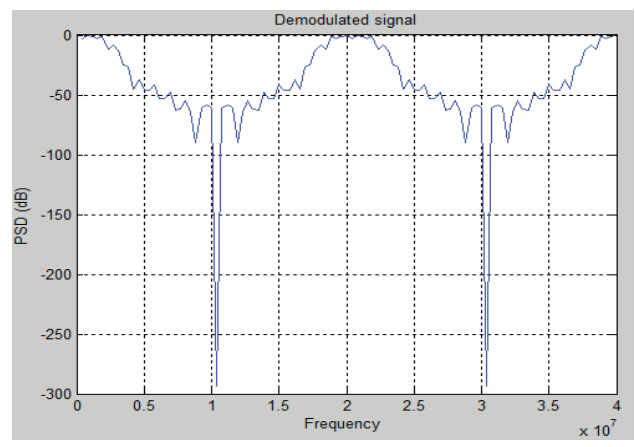

Figure 11. Demodulated signal.

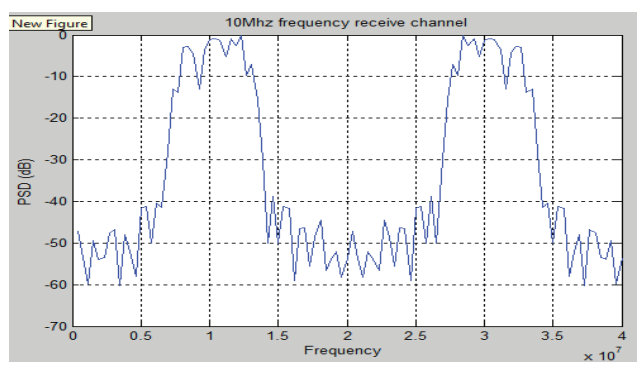

Figure 12. Carrier signal frequency in receiver side.

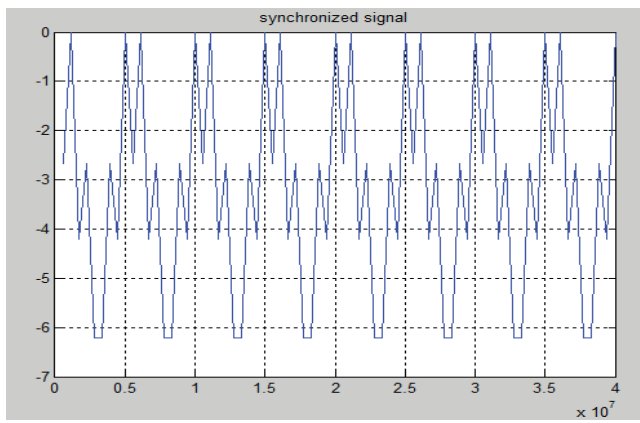

Figure 13. Synchronized signal.

synchronize the carrier signal frequencies of transmitter and receiver, and to minimize the multipath effects (Figure 13).

From the Figures 5 and 13 it is observed that the carrier synchronization has been achieved $[5,6]$.

\section{Conclusion}

The software defined radio offers the flexibility and simultaneously providing high performance to attain the different kinds of modulation standards in the upcoming generations in the communication field. In the communication field synchronization of carrier signal is one of the challenging tasks. In this paper for transmission of data or message signal 8PSK modulation scheme is used. Carrier recovery has been performed using Costas loop.

\section{References}

1. Dick C, Harris F et al. (2000). Synchronization in software radios-carrier and timing recovery using FPGAs, Field-Programmable Custom Computing Machines, 2000 IEEE Symposium, 195-204.

2. Rodriguez A S, Mensinger M M et al. (2011). Model-based Software-defined Radio (SDR) design using FPGA, Electro/ Information Technology (EIT), 2011 IEEE International Conference, 1-6.

3. Alluri V B, Heath J R et al. (2010). A new multichannnel, coherent amplitude modulated, gate array technology implementation, IEEE Transaction on Signal Processing, vol 58(10), 5369-5384.

4. Katz S, and Flynn J (2009). Using Software Defined Radio (SDR) to demonstrate concepts in communications and signal processing courses, Frontiers in Education Conference, $1-6$.

5. Zhang B (2009). Real-time software-defined-radio implementation of time-slotted carrier synchronization for distributed beamforming, Chapter 2, 10-14.

6. Husted P J. Design and implementation of digital timing recovery and carrier synchronization for high speed wireless communications, Research Project, Chapter 3, 37-40.

7. Mileant A, and Hinedi S (1989). Costas loop lock detection in the advanced receiver, TDA Progress Report, 72-89. 